\title{
Les lettres de Jacques Godechot à Albert Manfred et aux responsables de l'Annuaire d'études françaises
}

\section{Varoujean Poghosyan}

\section{(2) OpenEdition \\ 1 Journals}

\section{Édition électronique}

URL : https://journals.openedition.org/ahrf/13513

DOI : $10.4000 /$ ahrf.13513

ISSN : 1952-403X

Éditeur :

Armand Colin, Société des études robespierristes

\section{Édition imprimée}

Date de publication : 1 juin 2015

Pagination : 133-147

ISBN : 9782200929718

ISSN : 0003-4436

\section{Référence électronique}

Varoujean Poghosyan, «Les lettres de Jacques Godechot à Albert Manfred et aux responsables de I'Annuaire d'études françaises », Annales historiques de la Révolution française [En ligne], 380 l juin 2015, mis en ligne le 01 juin 2018, consulté le 24 avril 2022. URL : http://journals.openedition.org/ahrf/13513 ; DOl : https://doi.org/10.4000/ahrf.13513 


\title{
LES LETTRES DE JACQUES GODECHOT À ALBERT MANFRED ET AUX RESPONSABLES DE L'ANNUAIRE D'ÉTUDES FRANÇAISES
}

\author{
Varoujean POGHOSYAN
}

Au mois d'octobre 2012, j' ai eu la possibilité de travailler dans les archives personnelles d'Albert Manfred (1906-1976), l'un des plus éminents historiens soviétiques. Leur richesse m'a tout simplement stupéfait ; parmi ses innombrables correspondants figuraient non seulement des historiens soviétiques et étrangers mais également d'éminents écrivains soviétiques et des hommes politiques français, comme Maurice Thorez, Georges Cogniot, Jacques Duclos ${ }^{1}$. De toutes ces relations, celles entretenues avec les Français ont peut-être été les plus constantes. Le fonds d'archives contient de la correspondance entre Albert Manfred et Fernand Braudel, Albert Soboul, Claude Willard, Jean-Baptiste Duroselle, Jean Bruhat et beaucoup d'autres. Pour l'historien de la Révolution, les lettres de Jacques Godechot (1907-1989) se distinguent par leur grand intérêt.

J'avais commencé à lire les études d'Albert Manfred sur la Révolution française et le Premier empire lors de mes études en deuxième année de l'Université d'État d'Erevan. J'avoue que c'est son œuvre qui m'a fait découvrir la Révolution française, et m'a incité, après mes études universitaires, à consacrer mes recherches aux problèmes de l'époque révolutionnaire. À vrai dire, étant encore étudiant, je n'avais qu'un seul rêve, celui de continuer mes recherches dans ce domaine sous sa direction. C'est pourquoi, la quatrième année à peine terminée (à cette époque, la formation

(1) Dans les archives d'Albert Manfred est conservée aussi la copie tapée à la machine de sa lettre à Edgar Faure. Celle-ci n'est pas datée mais, d'après son contenu, on peut constater qu'elle a probablement été écrite au milieu des années 1970. Il s'agissait dans cette lettre de la traduction russe de son ouvrage sur La Disgrâce de Turgot dont Albert Manfred était l'ordonnateur (voir Département scientifique des manuscrits de la Bibliothèque d'État russe, fonds 772, carton 40, dossier 17, p. 1). Ce livre a été publié à Moscou aux éditions du Progrès en 1979 dans une rédaction de Victor Daline. 
se déroulait durant cinq ans), je suis parti pour Moscou dans le but de le rencontrer. Par un curieux hasard, j' ai eu avec lui une seule rencontre, le 14 juillet 1976, à l'Institut d'histoire universelle de l'Académie des sciences de l'URSS, où il travaillait; c'était cinq mois avant son décès. Dès qu'il a compris pourquoi j'étais arrivé d'Erevan à Moscou, il s'est tourné vers moi et a commencé à parler en français. Après m'avoir posé nombre de questions, il a consenti à devenir mon futur maître. Cependant, le 16 décembre, quatre jours avant sa participation aux élections de l'Académie soviétique, dont les membres rejetaient toujours sa candidature, il est décédé dans l'un des hôpitaux de Moscou. Ce fut juste après son décès que les revues et journaux soviétiques l'ont qualifié de «plus grand historien » et de «plus grand savant » soviétique. C'était la réalité soviétique. J'ai continué mes études à l'Institut d'histoire universelle, à partir de 1978, sous le patronage de Victor Daline, son meilleur ami.

À la différence d'Albert Manfred je n'ai jamais eu le plaisir de rencontrer Jacques Godechot car, en ex-URSS, les jeunes chercheurs n'avaient pas le droit de partir en mission scientifique pour les pays «capitalistes ». Albert Soboul, dont j'avais eu la chance de faire la connaissance au mois de juin 1978, lors de son séjour à Moscou pour prendre part aux travaux du VIII ${ }^{\mathrm{e}}$ Colloque des historiens de l'URSS et de la France, a bien tenté de m'inviter à la Sorbonne, mais en vain. Juste après son arrivée, j'ai pu discuter avec lui à l'hôtel de l'Académie soviétique, où je lui ai précisé que je m'occupais de l'histoire politique du premier Directoire. Après quelques échanges, il a conclu : «Le Directoire est un bon sujet, mais il faut que vous travailliez à Paris. Je vous enverrai un visa personnel, mais vous devez travailler avec Suratteau, et non pas avec moi ».

Je n'ai pu davantage rencontrer Jacques Godechot, mais j'ai pu entretenir une correspondance suivie avec lui à partir de 1982. Dès le début de ma carrière scientifique, à partir de janvier 1978, j'étais déjà initié à son œuvre majeure. Sur les conseils de Victor Daline, j'avais commencé à étudier l'histoire du Directoire par les livres d'Albert Mathiez et de Georges Lefebvre et, quand j'ai ouvert dans l'une des bibliothèques moscovites Le Directoire de Mathiez, j' ai aperçu pour la première fois le nom de Jacques Godechot, qui avait pris soin de publier ce livre après la mort de son maître ${ }^{2}$.

(2) On a, à tort, récemment attribué le travail réalisé par Jacques Godechot à Georges Lefebvre : voir Christian DELACROIX, François DosSE et Patrick GARCIA, Les courants historiques en France $X I X^{e}-X X^{e}$ siècle, Paris, Gallimard, 2009, p. 180. 
Ses livres, ses articles historiographiques dans la Revue historique et ses recensions innombrables dans les $A H R F$ ont laissé une empreinte ineffaçable sur moi. Ils m'ont également beaucoup aidé à m'orienter dans le domaine le plus difficile de la science historique, celui de l'historiographie. J'ai également été frappé par son indépendance d'esprit ; il n'a jamais adhéré à aucun parti, ni à aucune « école » historique ; cela m'a beaucoup séduit ${ }^{3}$. Aujourd'hui, sa conduite ne m'étonne plus, car il avait été l'élève d'Albert Mathiez, qu'il a plus tard qualifié de «paysan du Danube $»^{4}$.

Au début des années 1980, Victor Daline m'a conseillé d'envoyer à Jacques Godechot les tirés-à-part de mes premiers articles sur le Directoire, ce que j'ai fait. Et ce grand historien répondait sans tarder, à chacun de mes envois, à chacune de mes lettres, en me remerciant, en m'encourageant, ainsi qu'en approuvant ma collaboration possible avec les $A H R F$. Il exprimait aussi des jugements impartiaux à propos de mes articles, d'après leurs résumés en français, qu'il lisait, comme il l'avouait, «avec plaisir », précisant en même temps, qu'il était déjà au courant de mes études après les avoir lues dans les volumes de l'Annuaire d'études françaises, qu'il possédait grâce à Victor Daline. Si nécessaire, Jacques Godechot discutait avec moi de différents problèmes de l'époque directoriale, ce dont je lui étais très reconnaissant, et parfois, il me donnait des conseils toujours pertinents. En 1988, quand notre correspondance concernait son livre sur Le comte d'Antraigues, il me conseilla dans sa lettre du 20 juin, de chercher dans les archives soviétiques des documents sur l'action du comte d'Antraigues : « Ainsi que vous avez pu le constater, écrivait-il, je n'ai fait, moi-même, aucune recherche dans les archives russes, je m' appuie sur celles de Pingaud et de Bouloiseau. Mais je suis persuadé qu'il existe dans les archives soviétiques encore de nombreuses lettres du comte d'Antraigues, puisqu'il a été au service de la Russie de 1793 jusqu'à sa mort en 1812. La découverte de ces lettres pourrait compléter et sans doute modifier le portrait que j'en ai fait $»^{5}$. Dans ces conditions et en dépit de la distance existant entre nous, j'ai pu considérer Jacques Godechot comme l'un de mes maîtres, au même titre qu'Albert Manfred et Victor Daline ${ }^{6}$.

(3) «Préface » dans Jacques GODECHOT, Regards sur l'époque révolutionnaire, Toulouse, Privat, 1980, p. 9-10 ; Claude PETITFRÈRE, « Jacques Godechot (1907-1989) », AHRF, 1990, n² 281, p. 317. Claude Petitfrère rappelle que Jacques Godechot lui a « enseigné deux attitudes d'esprit fondamentales : dans la recherche de l'écriture de l'histoire, la rigueur et l'honnêteté, dans la vie, la tolérance et le prix de la liberté ». Voir Claude PETITFRÈRE, op. cit., p. 317.

(4) Jacques GoDECHOT, Un jury pour la Révolution, Paris, Robert Laffont, 1974, p. 301.

(5) Les archives personnelles de l'auteur.

(6) J'ai dédié à sa mémoire mon livre Parmi les historiens, en russe et en français, Erevan, Édit Print, 2011. 
Victor Daline estimait profondément et sincèrement Jacques Godechot, avec qui il se trouvait, à son tour, en correspondance : il l'avait vu seulement deux fois, lors de deux visites de son collègue français en URSS, en 1969 et en 1970, quand le doyen de Toulouse y était venu pour participer aux travaux du $\mathrm{IV}^{\mathrm{e}}$ Colloque des historiens de l'URSS et de la France à Erevan, et à ceux du XIII ${ }^{\mathrm{e}}$ Congrès international des historiens de Moscou. La sortie de Victor Daline de l'URSS était interdite car il avait été l'une des victimes de la «terreur stalinienne », ayant passé les meilleures années de sa vie (presque dix-sept ans) dans les camps staliniens. D'ailleurs, étant l'un des fondateurs de la science historique soviétique, il n'imaginait point l'interprétation de l'histoire de la Révolution française hors de la méthodologie marxiste. Cependant, quand je répétais en l'occurrence et sans aucune hésitation, que je considérais Jacques Godechot comme l'un de mes maîtres, il approuvait ma prise de position.

Victor Daline envoyait constamment ses livres et ses articles, ainsi que les livres de ses collègues soviétiques, à Jacques Godechot. Et l'historien français regrettait toujours dans ses réponses de ne pas maitriser la langue russe. Il m'écrivait bien souvent la même chose, parfois répétant à ce sujet, presque littéralement, les mots d'Albert Mathiez. Par la lecture des résumés français des livres et des articles de ses collègues soviétiques, il demeurait cependant au courant des recherches historiques en URSS.

Certes, les historiens soviétiques ne partageaient pas entièrement les vues de Jacques Godechot, surtout sa théorie de la « révolution atlantique », mais en dépit de cela, ses livres lui ont assuré un vrai succès en ex-URSS. D'ailleurs, la majorité des historiens soviétiques de la Révolution française, dont Anatoli Ado, reconnaissaient que l'approche de Jacques Godechot à l'égard de l'époque révolutionnaire était, dans son ensemble, assez proche de celle des historiens marxistes. C'était notamment l'attitude de Victor Daline, qui a présenté les Regards sur l'époque révolutionnaire aux lecteurs de l'Annuaire d'études françaises ${ }^{7}$. Albert Manfred, opposant farouche de l'idée d'une "révolution atlantique », ne s'est quant à lui jamais permis, à la différence de quelques-uns de ses collègues soviétiques, de qualifier Jacques Godechot d' " historien bourgeois », même au milieu des années 1960, quand la critique de cette théorie avait atteint son apogée. Il considérait au contraire Jacques Godechot comme « le plus grand spécialiste

(7) Victor DALINE, «Sur le jubilé de Jacques Godechot», en russe, Annuaire d'études françaises - 1981, Moscou, Naouka, 1983, p. 253-254. 
des problèmes de l'histoire de la Révolution française, dont le nom faisait autorité et qui jouissait d'un respect mérité $»^{8}$. En maintes occasions, il a qualifié ses Commissaires aux armées sous le Directoire et sa Prise de la Bastille comme des études « exemplaires » et « remarquables $»^{9}$.

Anatoli Ado s'est rappelé lors d'une conversation avec moi en 1983, de l'accueil bienveillant que Jacques Godechot lui avait montré lors de l'une de ses missions scientifiques en France. Les historiens américains, en rendant un profond hommage à sa mémoire, ont également constaté son amabilité, n'oubliant pas de citer son attitude cordiale envers les chercheurs allemands, même après la guerre ${ }^{10}$. Je peux également attester de cette courtoisie, jamais démentie ${ }^{11}$; il m'envoyait les tirés-à-part de ses articles avec d'aimables dédicaces.

N'étant point un historien marxiste, Jacques Godechot appréciait pourtant à leur juste valeur l'œuvre des historiens soviétiques, dont les recherches se trouvaient toujours au centre de son attention. Ce n'était pas par hasard qu'il écrivait à ses collègues dans sa lettre du 22 avril 1969, après la réception de l'Annuaire d'études françaises de 1967 : «Je vois que vous réalisez une œuvre considérable, dont je vous félicite ». Il publiait régulièrement, soit dans la Revue historique, soit dans les $A H R F$, des recensions sur les traductions des articles et surtout des livres de ses confrères soviétiques, de ses collègues soviétiques, et en premier lieu sur ceux d'Albert Manfred et de Victor Daline. En dépit de sa divergence d'opinions, surtout avec Albert Manfred, il l'estimait profondément, et les lettres qu'il lui adressait et que nous présentons aux lecteurs de notre revue, en sont la meilleure preuve. Je me rappelle bien l'une des dédicaces qu'il avait faite sur son livre L'Europe et l'Amérique à l'époque napoléonienne que j'ai feuilleté jadis dans la bibliothèque personnelle d'Albert Manfred : « $\mathrm{Au}$ grand historien russe Albert Manfred». De son propre avis, le Napoléon Bonaparte de Manfred méritait « d'être placé parmi les meilleures

(8) Il s'agit de son discours «Sur certaines questions controversées et non résolues de l'historiographie de la Révolution française », présenté au VII ${ }^{\mathrm{e}}$ Colloque des historiens de l'URSS et de la France, dont les travaux se sont déroulés à Paris et à Dijon. Voir ce texte publié en russe dans l'Annuaire d'études françaises - 1976, Moscou, Naouka, 1978, p. 173. 1980 , p. 131

(9) Ibidem, p. 172 ; Albert MANFRED, Napoléon Bonaparte, en français, Moscou, Éd. Progrès,

(10) «American Historians Remember Jacques Godechot. Robert Forster, Robert. R. Palmer, James Friguglietti, and Emmet Kennedy », French Historical Studies, 1990, v. 16, n 4, p. 879-892.

(11) Il y a lieu, comme il me semble, de me référer à Georges Fournier, qui précise que Jacques Godechot « avait pour chacun un sourire amical, quelques mots d'encouragement ou quelques conseils pertinents » et « qu'il se dégageait de sa personne une sérénité qui semblait effacer tous les soucis du moment ». Voir Georges FOURNIER, « Jacques Godechot et le Midi toulousain », AHRF, 1990, n $^{\circ} 281$, p. 328. 
biographies de l'empereur $»^{12}$. Et ce n'est point étonnant qu'il ait critiqué Gilbert Comte, l'un des journalistes français, d'avoir ridiculisé son collègue et ami soviétique en traitant dans le Monde du 16 mai 1982 ce livre de « Napoléon soviétique ${ }^{13}$.

Jacques Godechot a ainsi permis l'existence de cordiales et fructueuses relations entre les historiens soviétiques et français, et il entretenait des relations très amicales avec Victor Daline et Guennadi Koutcherenko. Il participait aux colloques des historiens soviétiques et français, ne refusait jamais de publier ses articles dans les éditions soviétiques ${ }^{14}$, et il invitait constamment Albert Manfred à l'université de Toulouse, pour y donner des conférences. Albert Manfred écrivait, en 1976 : « J'ai un grand respect pour le professeur Godechot, fréquent participant de nos colloques, et dont nous apprécions tous hautement la contribution personnelle au développement de la coopération scientifique entre les historiens de nos deux pays $»^{15}$.

Je ne doute pas que les lettres de Jacques Godechot à Albert Manfred et aux responsables de l'Annuaire d'études françaises contribuent à mieux connaître les relations entre les historiographies soviétiques et françaises des années 1960 et 1970. Qu'il me soit permis de préciser, pour conclure, que les archives personnelles d'Albert Manfred se trouvent dans le Département scientifique des manuscrits de la Bibliothèque d'État russe à Moscou (fonds 772). Les lettres de Jacques Godechot, que nous publions, adressées en premier lieu à Albert Manfred et, partiellement, aux responsables de l'Annuaire d'études françaises, à l'exception de celle du 8 janvier 1966, sont tapées à la machine (fonds 772, carton 43, dossier 39).

\section{1}

Paris, le 8 janvier 1966

[de Paris à Paris]

(12) Jacques GODECHOT, « La période révolutionnaire et impériale (publications de 1978 à $1985) »$, Revue historique, 1986, t. 557, p. 200.

(13) Voir sa recension sur la traduction française de ce livre dans les $A H R F, \mathrm{n}^{\circ} 249$, p. 495.

(14) Jacques GODECHOT, «État actuel des études sur la Révolution française dans le monde Occidental », en russe, Annuaire d'études françaises - 1970, Moscou, Naouka, 1972, p. 264-277 ; idem, «Sur le livre de François Furet », en russe, Annuaire d'études françaises - 1979, Moscou, Naouka, 1981, p. 255-260; id., «L'expansion révolutionnaire en Europe et en Amérique » dans $L a$ Grande Révolution française et la Russie, Moscou, Progrès, 1989, p. 82-90 (ce recueil d'articles et de documents a vu le jour à Moscou en 1989 non seulement en russe, mais aussi en français).

(15) Voir Albert MANFRED, «Sur certaines questions controversées et non résolues de l'historiographie de la Révolution française », p. 172. 
Mon cher Collègue,

Je n'ai peut-être pas assez insisté ce matin pour vous dire à quel point la Faculté des Lettres de Toulouse serait heureuse que vous veniez y faire une ou deux conférences. Je me permets de vous précisiez que, si vous vous décidez, la Faculté prendra à sa charge vos frais de voyage et du séjour. J'espère, en tout cas, que si vous ne pouvez venir maintenant, vous ne manquerez pas d'inscrire Toulouse dans votre prochain programme de voyage.

Veuillez croire, mon cher collègue, à mes sentiments les plus cordialement dévoués.

[signature]

Jacques Godechot

\section{2}

Toulouse, le 10 août 1966

Mes chers collègues,

Je vous remercie de m'avoir envoyé, avec une aussi aimable dédicace l'Annuaire d'études françaises ${ }^{16}$ que vous venez de publier. J'ai lu avec un vif intérêt les résumés en français des différents articles, et j'en ai tiré grand profit. Je remercie tout particulièrement $\mathrm{M}$ [onsieur] Daline pour son article sur Gilbert Romme et Pavel Stroganov ${ }^{17}$. Il apporte des compléments inédits et fort intéressants à ce que nous savions de l'influence exercée hors de France par la Révolution.

En attendant que nous ayons l'occasion de nous rencontrer, je vous prie de croire, Mes Chers Collègues, à mes sentiments les plus cordiaux.

[signature]

Jacques Godechot

\section{3}

Toulouse, le 7 février 1967

Mon cher collègue,

(16) Paru en 1967.

(17) Voir Victor DaLINE, « Gilbert Romme, Pavel Stroganov et la cour de Saint-Pétersbourg », Gilbert Romme (1750-1795) et son temps. Actes du Colloque tenu à Riom et Clermont (10-11 juin 1965), Paris, PUF, 1966, p. 69-80. 
Je vous remercie beaucoup d'avoir eu l'amabilité de m'envoyer votre ouvrage sur « La Grande Révolution française » ${ }^{18}$. Il me paraît extrêmement intéressant et original. Je vais le lire avec le plus grand plaisir ${ }^{19}$.

J'espère avoir bientôt l'occasion de vous rencontrer. Je souhaite surtout qu'à votre prochain voyage en France, vous puissiez venir jusqu'à Toulouse, et en attendant, je vous prie de croire à mes sentiments les meilleurs.

[signature]

Jacques Godechot

\section{4}

Toulouse, le 14 avril 1967

Mon cher collègue,

Je vous remercie beaucoup de m'avoir envoyé l'annuaire des études historiques de $1965^{20}$. J'ai lu avec plaisir le résumé français des études relatives à la Révolution. L'une de celles-ci a été publiée dans les Annales historiques de la Révolution française ${ }^{21}$. Il est souhaitable que d'autres puissent également, en entier, être publiées en traduction française.

En attendant le plaisir de vous revoir, croyez, mon cher collègue, à mes sentiments les meilleurs.

[signature]

Jacques Godechot

(18) Il s'agit de la traduction française du livre d'Albert Manfred sur La Grande révolution française du XVIII ${ }^{e}$ siècle, parue à Moscou en 1961 dans les Éditions en langues étrangères. Voir le compte rendu de Jacques Godechot sur ce livre dans la Revue historique (1972, t. 504, p. 479).

(19) Les deux éditions de ce livre d'Albert Manfred, publiées à Moscou en 1950 et 1956, ont été traduites en huit langues et publiées dans différents pays. Certes, il s'agit d'un livre de vulgarisation, rédigé sur la base de la méthodologie marxiste, or sa portée incontestable dans l'éducation de beaucoup de générations d'historiens de la Révolution est évidente. Walter Markov, par exemple, avait constaté : «Dans les Universités de beaucoup de pays socialistes, le travail d'Albert Z. Manfred est devenu le premier livre pour les séminaires, lors de la préparation des examens de la jeune génération montante des historiens de la Révolution française ; on ne pouvait presque pas rencontrer un étudiant qui n'avait pas utilisé ce travail, qui ne s'était pas orienté d'après lui ». Walter MARKOV, « A. Z. Manfred, historien de la Grande Révolution française », en russe, Annuaire d'études françaises - 1981, Moscou, Naouka, 1983, p. 155.

(20) Il fait une allusion à l'Annuaire d'études françaises - 1965, paru en 1966.

(21) Il m'est difficile de préciser de quel article il s'agit. Dans ce volume de l'Annuaire furent publiés seulement deux articles sur l'époque révolutionnaire, celui de Walter MARKOV, « Jacques Roux et Karl Marx », en russe, p. 66-75, et celui d'Anatoli ADO « Sur la pétition présentée par Pierre Dolivier à l'Assemblé Législative le 1 mai 1792 », en russe, p. 229-232. 
Mon cher Collègue,

Je vous envoie aujourd' hui-même mes trois derniers livres : L'Europe et l'Amérique à l'époque napoléonienne, La Prise de la Bastille et un petit essai sur Talleyrand publié en Italie ${ }^{22}$.

Je joins à mon envoi un certain nombre de tirages à part qui me restent $^{23}$. Ce sont des articles parus à des époques différentes, certains il y a déjà bien longtemps. Peut-être trouverez-vous parmi ces articles une étude que vous jugerez digne de publier dans l'Annuaire d'Histoire de votre Institut ${ }^{24}$.

Je regrette que cette fois encore vous n'ayez pu venir jusqu'à Toulouse, mais j'espère qu'à votre prochain voyage il vous sera possible de pousser jusqu'à notre ville. Moi-même je serais très heureux de me rendre en Union Soviétique ainsi que vous me l'avez proposé.

En attendant le plaisir de vous y rencontrer, je vous prie de croire, Mon cher Collègue et Ami, à mes sentiments les plus cordialement dévoués.

[signature] Jacques Godechot

\section{6}

Toulouse, le 22 avril 1969

Messieurs les professeurs Manfred, Daline, Jeloubovskaia ${ }^{25}$

Mes chers Amis,

Je vous remercie beaucoup de m'avoir envoyé avec une si aimable dédicace l'Annuaire d'études françaises pour $1967^{26}$.

(22) Jacques GoDECHOT, Talleyrand, Milano, C.E.I., 1966.

(23) Dans les archives d'Albert Manfred sont conservés les tirés-à-part des articles suivants de Jacques Godechot : Jacques GODECHOT, « La création de la Première république Italienne (1802) », Estratto da Quaderni Francesi, vol. 1, Napoli 1970 ; idem, « Originalité et imitation dans les institutions Italiennes de l'époque napoléonienne », Astratto dai volumi XXIII-XXIV (1971-1972) dell' Annuario dell' Institutio Storico Italiano per l'età moderna e contemporenea, Roma 1975 ; id., « Les Français et l'unité Italienne sous le Directoire », Revue d'histoire politique et constitutionnelle, 1952. Voir Département scientifique des manuscrits de la Bibliothèque d'État russe, fonds 772, carton 57, dossier 24.

(24) Il s'agit de l'Annuaire d'études françaises.

(25) Enna Jeloubovskaya, ancienne membre du comité de la rédaction de l'Annuaire d'études françaises, spécialiste du Second empire.

(26) Ce volume a vu le jour en 1968. 
J'ai lu avec un vif intérêt les résumés français des communications qui ont été présentées en russe. Je vois que vous réalisez une œuvre considérable, dont je vous félicite.

Je vous prie de croire, mes chers Amis, à l'assurance de mes sentiments les plus cordiaux.

[signature]

Jacques Godechot

\section{7}

Toulouse, le 23 décembre 1969

Mon cher Collègue,

À mon retour de Russie j'ai été extrêmement occupé par de très nombreuses obligations. C'est seulement maintenant, à la fin de l'année, que je puis songer aux promesses que je vous ai faites. Je vous envoie donc par le même courrier, mon livre sur Napoléon, un recueil sur « La Pensée révolutionnaire en Europe de 1780 à 1789 », une petite brochure en italien sur Talleyrand qui est imprimée en même temps qu'une étude sur Napoléon par Walter Markov, et un travail que j'ai dirigé sur l' « Évolution démographique de Toulouse au $18^{\mathrm{e}}$ siècle $»^{27}$.

Je n'ai pas encore eu le temps de rédiger le rapport que j'ai présenté à Erivan $[\mathrm{sic}]^{28}$. Je pense mettre à profit les vacances pour le faire, et vous l'adresser dans une dizaine de jours.

Croyez, mon cher Collègue, à l'assurance de mes sentiments les plus cordiaux et à mes vœux les meilleurs pour la nouvelle année.

[signature]

Jacques Godechot

\section{8}

Toulouse, le 4 février 1970

Mon cher Collègue et Ami,

Je vous envoie, ci-joint, avec un retard dont je vous prie de m'excuser, le texte de la communication que j' ai présenté au Colloque franco-soviétique d'Erivan [sic].

(27) Voir Jacques GoDECHOT, «L'histoire sociale et économique de Toulouse au XVIII ${ }^{\mathrm{e}}$ siècle », Annales du Midi, 1966, p. 363-374.

(28) Il s'agit du IV e Colloque des historiens de l'URSS et de la France qui a eu lieu à Erevan en novembre 1969. 
Je vous serais très obligé de bien vouloir me faire savoir dans quelle revue vous avez l'intention de le publier. Est-ce dans l'Annuaire d'études françaises? Vous m'aviez demandé aussi de vous adresser un article publié antérieurement dans une revue historique française et dont la traduction russe pourrait paraître dans Annuaire d'études françaises. Je vous envoie, ci-joint, une étude sur « Les Français et l'unité italienne sous le Directoire » qui a été publiée il y a une quinzaine d'années dans la Revue d'histoire politique et constitutionnelle, aujourd'hui disparue, et en traduction italienne dans la Rivista storica italian ${ }^{29}$. Je crois que cet article pose des problèmes très importants, de nature à intéresser les historiens soviétiques. Je l'ai revu et j'en ai complété les notes. cordiaux.

Croyez, mon cher Collègue et Ami, à mes sentiments les plus [signature]
Jacques Godechot

9

Toulouse, le 10 avril 1970

Mon cher Ami,

Je vous remercie beaucoup de m'avoir envoyé votre livre d'études sur l' « Histoire de la France » et sur «Les rapports entre la France et l'Union Soviétique $\gg^{30}$. Il est très heureux que vos différents articles aient été réunis en volume. Je suis certain que cet ouvrage rencontrera un vif succès auprès des historiens français ${ }^{31}$.

(29) Jacques GoDECHOT, «Les Français et l'unité italienne sous le Directoire », Revue d'histoire politique et constitutionnelle, 1952, p. 548 -580; idem, « Francesi e l'unità italiana sotto il Direttorio », Rivista storica italiana, 1952, p. 96-110, 193-204. Cet article n'a pas été publié en version russe.

(30) Il s'agit de la traduction française du livre d'Albert Manfred, Essai d'histoire de France du XVIII ${ }^{e}$ au XX $X^{e}$ siècle. Recueil d'articles, publié à Moscou par les Éditions du Progrès en 1969.

(31) Jacques Godechot avait raison : voir les recensions de Jean Bruhat (France-URSS, 1970, $\mathrm{n}^{\circ}$ 25, p. 30), de Madeleine Rebérioux (Annales, 1971, $\mathrm{n}^{\circ}$ 5, p. 1077), de Jacques Godechot (Revue historique, 1972, t. 504, p. 479), de Maurice Agulhon (Mouvement social, 1975, nº 92, p. 119-122). Voir aussi celle de Walter Markov dans la revue Zeitschfrift für Geschichtswissenschaft, 1972, H. 2, S. 240-241. Notons, toutefois, que les interprétations d'Albert Manfred non pas été entièrement partagées par tous ses collègues français. Jean-Baptiste Duroselle, par exemple, après la réception de ce livre lui écrivait : «Merci infiniment de m'avoir envoyé ce précieuse [sic] recueil de certains de vos travaux concernant la France. J'ai aussitôt commencé à le lire. Je connaissais déjà, évidemment, certains textes, mais je les relis avec plaisir. Il y a certes des points sur lesquels je ne partage pas entièrement votre interprétation. Mais, dans l'ensemble nous ne sommes pas éloignés. Et j'ai beaucoup apprécié votre conclusion ». Voir Département scientifique des manuscrits de la Bibliothèque d'État russe, f. 772, c. 42, doss. 46, p. 4. 
En attendant le plaisir de vous revoir à Moscou cet étée ${ }^{32}$, je vous prie de croire, mon cher Ami, à mes sentiments les plus cordiaux.

[signature]

Jacques Godechot

10

Toulouse, le 6 avril 1972

Cher Ami,

Je vous remercie beaucoup de m'avoir envoyé votre livre d'études sur « Napoléon Bonaparte $»^{33}$. Comme malheureusement j'ignore la langue russe, je n'ai pas pu le lire, mais j'ai vu en feuilletant l'index et en me reportant aux notes que vous aviez cité plusieurs de mes ouvrages. J'espère que ce livre sera prochainement traduit en français ${ }^{34}$ et que $\mathrm{j}$ ' aurai le plaisir de le lire ${ }^{35}$. En attendant,

Je vous prie de croire, cher Ami, à l'assurance de mes sentiments les plus cordiaux.

[signature]

Jacques Godechot

11

Toulouse, le 18 août 1972

Mes chers Collègues et Amis,

Je viens de recevoir l'Annuaire d'Études françaises de $1970^{36}$ que vous m'avez si aimablement dédicacé et j'ai vu avec plaisir qu'il contient la traduction de la communication que j'ai présentée au Colloque francosoviétique d'Erivan [sic]. Je vous remercie beaucoup de l'avoir publiée ${ }^{37}$.

(32) Il fait une allusion à sa prochaine participation aux travaux du XIII ${ }^{\mathrm{e}}$ Congrès international des historiens qui a eu lieu à Moscou au mois d'août 1970.

(33) La première édition de ce livre a été publiée à Moscou par les Éditions du Misl en 1971.

(34) Remarquons que quelques-uns des collègues français d'Albert Manfred après la réception de ce livre lui ont posé la même question. Voir les lettres de Jean Bruhat du 14 avril 1972 (Département scientifique des manuscrits de la Bibliothèque d'État russe, f. 772, c. 42, doss. 42, p. 15) et de Claude Willard du 15 avril 1972 (Ibid., f. 772, c. 43, doss. 2, p. 16).

(35) Jacques Godechot a hautement apprécié ce livre après la lecture de sa traduction française, parue à Moscou dans les Éditions du Progrès en 1980 : voir ses deux recensions citées plus haut et publiées dans les AHRF (1982, n 249, p. 493-495) et dans la Revue historique (1986, t. 557, p. 199-200).

(36) Paru à Moscou en 1972.

(37) Jacques GODECHOT, «État actuel des études sur la Révolution française dans le monde Occidental », en russe, Annuaire d'Études françaises - 1970, Moscou, Naouka, 1972, p. 264-277. 
J'ai été aussi heureux de voir dans ce volume l'article de Madame Sokolova sur les revues historiques françaises contemporaines ${ }^{38}$, et le compte rendu du troisième Colloque sur l'histoire de la Révolution française, qui a eu lieu à Moscou en $1970^{39}$. Ces articles, et les autres aussi, font de ce volume un utile instrument de travail pour ceux qui désirent se mettre au courant des tendances actuelles de l'historiographie de la Révolution française. Par ailleurs, les articles sur Lénine et la France ${ }^{40}$ me paraissent (d'après leurs résumés en français), du plus haut intérêt.

En attendant le plaisir de vous revoir, je vous prie de croire à ma fidèle amitié.

[signature]

Jacques Godechot

12

Toulouse, le 5 janvier 1973

[de Toulouse à Paris]

Cher Ami,

Je vous remercie beaucoup de votre aimable lettre du 26 décembre.

Précisément aujourd'hui-même, le professeur de russe de notre Université, Madame Zamoyska m'a téléphoné qu'elle souhaitait que vous veniez à Toulouse donner une conférence aux étudiants de langue russe. Elle aimerait que vous leur parliez du thème de Napoléon dans la littérature russe. Un tel sujet intéresserait aussi un assez grand nombre d'étudiants d'Histoire. Moi-même, je souhaiterais que vous puissiez parler aux historiens de l'historiographie russe de la Révolution française.

Madame Zamoyska et moi nous espérons que vous pourrez venir à Toulouse dans la deuxième moitié de janvier, c'est-à-dire, plus précisément après le 16 janvier (car je serai absent le 15 et le 16), à une date de votre choix, mais de préférence un jeudi ou un vendredi.

Tous vos frais de voyage et de séjour seront bien entendu, à la charge de l'Université de Toulouse-Le-Mirail.

(38) Marianna SoKOLOVA, «Les revues historiques françaises contemporaines (aperçu historiographique) », en russe, ibidem, p. 244-266.

(39) Il s'agit du Colloque des historiens de la Révolution française qui a eu lieu le 19 août 1970, lors des travaux du XIII ${ }^{\mathrm{e}}$ Congrès international des historiens de Moscou. Voir « Le troisième Colloque sur l'histoire de la Révolution française », en russe, ibidem, p. 314. Lénine.

(40) La première partie de ce volume (p. 5-122) a été consacrée au centième anniversaire de 
Croyez, mon cher Ami, à l'assurance de mes sentiments les plus cordiaux, et à tous mes vœux pour 1973.

[signature]

Jacques Godechot

13

Toulouse, le 13 juin 1975

Mon cher Ami,

Je vous remercie beaucoup de m'avoir envoyé l'Annuaire d'études françaises $^{41}$ avec une si aimable dédicace. Heureusement pour moi, chaque article est suivi d'un bon résumé en français ce qui m'a permis de prendre connaissance des intéressants travaux publiés dans cette revue.

En attendant le plaisir de vous revoir bientôt, j'espère,

Croyez, mon cher Ami, à mes sentiments les plus cordiaux.

[signature]

Jacques Godechot

\section{4}

Toulouse, le 4 septembre 1975

Mon cher collègue et ami,

Je suis heureux de vous informer que la Commission internationale d'Histoire de la Révolution française qui s'est constituée le mardi 26 août 1975 à San Francisco vous a élu membre de son bureau ${ }^{42}$. Je vous en félicite et je vous adresse ci-joint le procès-verbal de la séance ${ }^{43}$. cordiaux.

Croyez, mon cher Collègue et ami, à mes sentiments les plus

Le président ${ }^{44}$,

[signature]

Jacques Godechot

(41) Il s'agit de l'Annuaire d'études françaises - 1973, paru en 1975.

(42) Albert Manfred a été élu président honoraire de cette Commission.

(43) Le fascicule de ce document est également conservé dans les archives d'Albert Manfred. Voir Département scientifique des manuscrits de la Bibliothèque d'État russe, f. 772, c. 43, doss. 39, p. 30-30 recto verso.

(44) Jacques Godechot a été élu président de cette Commission. 


\section{5}

Toulouse, le 17 août 1976

Cher ami,

J'apprends par Daline que vous allez avoir 70 ans le 28 août et je viens vous présenter à cette occasion mes vœux les plus cordiaux et les plus sincères de bon anniversaire. Moi-même, j'attendrai cet âge le 3 janvier prochain, nous sommes donc contemporains et je sais les avantages et les inconvénients qu'il comporte.

J'ai beaucoup regretté de ne pas vous avoir rencontré en mai dernier à Dijon. Mais ma femme devait précisément quitter l'hôpital et rentrer à la maison le jour du colloque de Dijon ${ }^{45}$, de sorte qu'il m'était tout à fait impossible de quitter Toulouse ce jour-là. Mon ancien élève et assistant Carbonell m'a dit qu'il avait été heureux de faire votre connaissance et m'a rapporté l'essentiel de ce qui a été dit au colloque.

J'espère que, malgré l'âge qui s'accroît, nous aurons l'occasion de nous rencontrer de nouveau ${ }^{46}$, et, en attendant, je vous prie d'accepter encore toutes mes félicitations et de croire à ma cordiale amitié.

[signature]

Jacques Godechot

Varoujean POGHOSYAN

Docteur es sciences historiques

Titulaire du Département des études génocidaires

Institut d'études arménologiques

Université d'État d'Erevan

Varuzhan.poghosyan@gmail.ru

(45) Il s'agit du VII ${ }^{\mathrm{e}}$ Colloque des historiens de l'URSS et de la France, dont les travaux se sont déroulés à Paris et à Dijon lors duquel Albert Manfred a présenté une communication sur certaines questions controversées et non résolues de l'historiographie de la Révolution française.

(46) Jacques Godechot a été privé de la possibilité de rencontrer à nouveau Albert Manfred. 